\title{
A propos d'un cas de méthémoglobinémie grave suite à une intoxication volontaire par une teinture de chaussures
}

\section{Case of severe methemoglobinemia following ingestion of a shoe dye}

\section{Bensalem BELLIK ${ }^{(1)}$, Khelifa DERGHAL ${ }^{(3)}$, Jacqueline SANCHO ${ }^{(2)}$, Abdelkader RHAOUI(4), Monique CAILLIEZ ${ }^{(2)}$, Anne-Marie DENANCE ${ }^{(3)}$, François FRAïSSE ${ }^{(4)}$}

(1) Laboratoire de Biologie des Urgences, Hôpital Pitié-Salpêtrière - 75013 PARIS - Tél : 0142177354

(2) Laboratoire de Biochimie-Immunologie, Hôpital Delafontaine - 93205 St-DENIS - Tél : 0142356140

(3) Urgences Médico-Chirurgicales, Hôpital Delafontaine - 93205 St-DENIS

(4) Service de Réanimation Médicale, Hôpital Delafontaine - 93205 St-DENIS

(Reçu le 21 février 2001 ; accepté le 29 avril 2001

\section{$\boldsymbol{R E} \boldsymbol{E} U \boldsymbol{M} \boldsymbol{E}$}

La méthémoglobinémie est une urgence thérapeutique dont le pronostic vital dépend de la précocité du diagnostic et du traitement spécifique (bleu de méthylène). Nous rapportons dans cette observation un cas de méthémoglobinémie grave (51\%) secondaire à l'ingestion d'une teinture de chaussures. Deux mois plus tard on assiste à une récidive avec le même produit. L'évolution sous bleu de méthylène a été nettement meilleure à la deuxième hospitalisation (taux de méthémoglobine $16 \%$ à la $2^{\text {ème }}$ heure sous $25 \mathrm{~mL}$ de bleu de méthylène versus $22 \%$ à la $24^{2 n t}$ heure sous $30 \mathrm{~mL}$ de bleu de méthylène) en raison d'une prise en charge plus efficace et plus rapide de la malade.

\section{MOTS-CLÉS}

Méthémoglobinémie, bleu de méthylène.

\section{SUMMARY}

Methemoglobinemia is a therapeutic emergency. Outcome depends on early diagnosis and specific therapy (by methylen blue). We here report a case of severe methemoglobinemia $(51 \%)$ following ingestion of a shoe dye; two month later a similar episode occured. Following therapy, a more favorable course was obtained during the second hospitalization (16\% after 2 hours with $25 \mathrm{~mL}$ methylen blue vs. $22 \%$ after 24 hours with $30 \mathrm{~mL}$ ). A more efficient care of methemoglobinemia relies on precocity of diagnosis and specific treatment.

\section{KEY-WORDS}

Methemoglobinemia, methylen blue. 


\section{Introduction}

La méthémoglobine correspond à l'oxydation du fer de l'hémoglobine. L'ion ferreux de l'hémoglobine perd un électron et devient ferrique $\left(\mathrm{Fe}^{++} \rightarrow \mathrm{Fe}^{+++}\right)$. Cette modification entraîne la diminution du contenu artériel en oxygène et la coloration acajou de l'hémoglobine à l'origine de la cyanose (1). A l'état physiologique le sang total ne doit pas contenir plus de $1 \%$ de méthémoglobine chez l'adulte, $1,5 \%$ chez le nouveau-né et $2 \%$ chez le prématuré (2). Au-delà de ce seuil, la fixation de l'oxygène est perturbée et l'hémoglobine devient incapable d'assurer une bonne oxygénation des tissus. Normalement, le globule rouge possède des systèmes réducteurs enzymatiques (fig. 1) qui assurent le maintien du taux de méthémoglobine au-dessous de la barre de $1 \%$. Le mécanisme le plus important est contrôlé par le système de la NADH-cytochrome-b5réductase (ou méthémoglobine-réductase-NADHdépendante), qui permet de capter des électrons du NADH pour réduire la méthémoglobine en hémoglobine. Le NADH est fourni par la voie principale de dégradation du glucose (ou voie anaérobie d'EmbdenMeyerhof qui représente $90 \%$ de la glycolyse intraérythrocytaire) (2). Ce système assure la réduction de $95 \%$ de méthémoglobine physiologique formée quotidiennement (bactéries nitrifiantes de l'intestin, produits méthémoglobinisants de l'alimentation).

Il existe un autre système moins important qui utilise le $\mathrm{NADPH}+\mathrm{H}^{+}$ou système de la NADPH-méthémoglobine-réductase. Le NADPH est fourni par la voie accessoire de la dégradation du glucose ou shunt accessoire des pentoses (la G6PD, glucose-6-phosphate-déshydrogénase est une enzyme clé de cette voie) (fig. 1). Le NADPH permet la réduction du bleu de méthylène en leucobleu, qui à son tour réduit la méthémoglobine en hémoglobine. Le rôle de ce système dans la réduction de la méthémoglobine en hémoglobine est modeste, car il nécessite un transporteur d'électrons qui n'est pas disponible dans l'organisme (vitamine $\mathrm{C}$ ou bleu de méthylène). En effet, en cas de méthémoglobinémie, les performances de cette voie augmentent significativement en présence de bleu de méthylène.

Les méthémoglobinémies acquises sont de loin les plus fréquentes, secondaires le plus souvent à une prise de toxiques, accidentelle ou volontaire. Les principaux toxiques méthémoglobinisants sont représentés dans le tableau I (3). Les formes congénitales peuvent être dues soit à des anomalies de structure de l'hémoglobine et favoriser la forme ferrique de l'hème (hémoglobines $\mathrm{M}$ ), soit à des déficits du système enzymatique (déficit en méthémoglobine réductase, en G6PD).

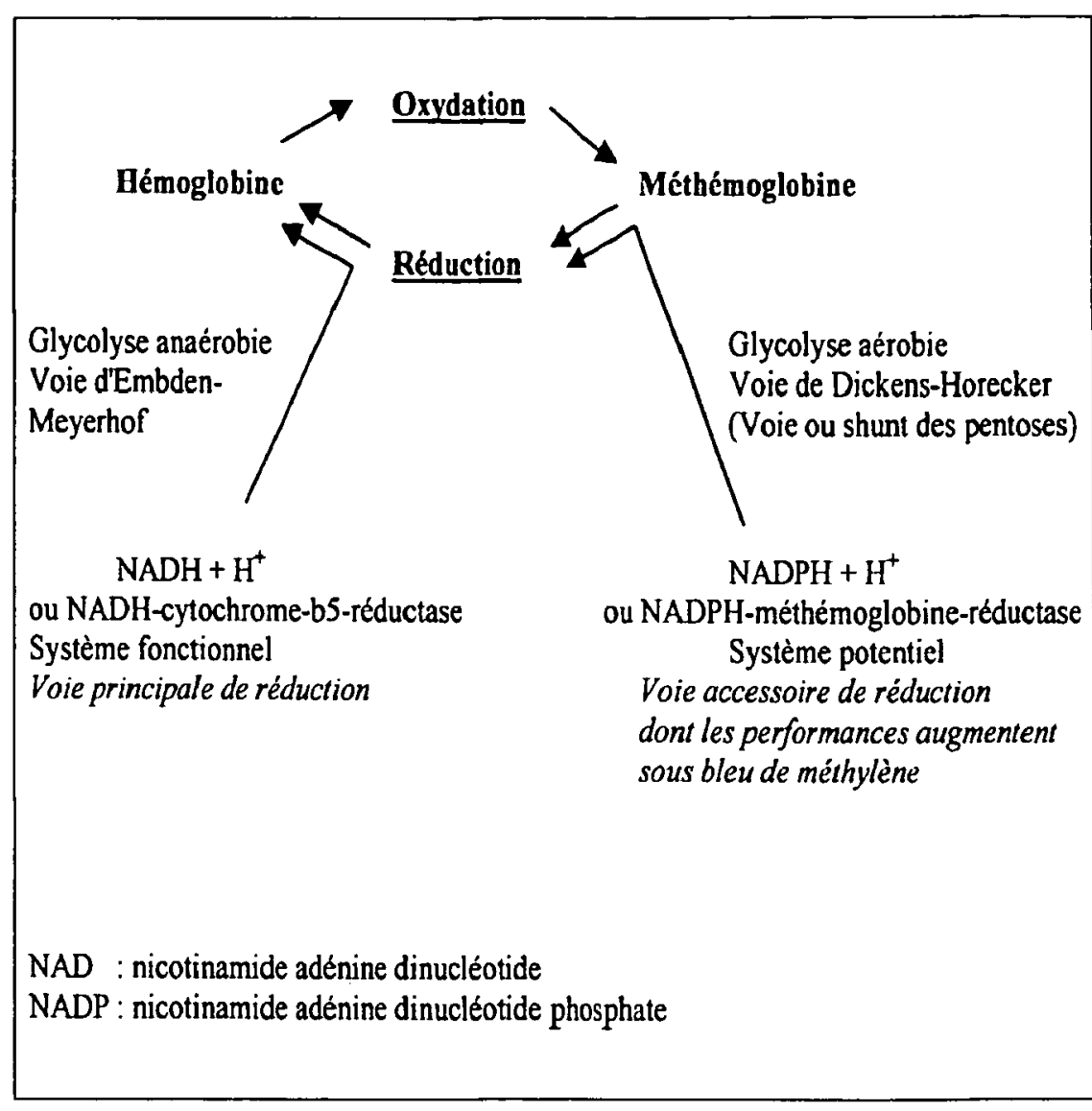

Figure 1 : Réduction de la méthémoglobine d'après DANEL V. et VIALA A. $(2,10)$.

Au-delà de 60-70\%, la méthémoglobinémie peut entraîner un coma ou un décès (4). Les signes cliniques, apparaissent en fonction de la gravité de la méthémoglobinémie (méthémoglobinémie par rapport à l'hémoglobine totale). A $10 \%$ apparaît la cyanose au niveau des doigts, du nez, des joues et des lèvres, à partir de $20 \%$ apparaissent céphalées, vertiges et tachycardie. Au-delà de 40-45 \% les signes sont plus graves : atteinte du système nerveux central, troubles de la conscience, collapsus, dépression respiratoire et arrêt cardiaque (5). Le tableau II présente les signes cliniques en fonction des taux de méthémoglobine.

La cyanose "gris ardoisé", généralisée, en l'absence de cause cardio-respiratoire et l'aspect brun foncé du prélèvement font évoquer une méthémoglobinémie (6). L'oxygénothérapie ne doit pas attendre le résultat du laboratoire. Elle consiste à administrer de l'oxygène à fort débit pour augmenter la quantité d'oxygène dissous dans la circulation sanguine et la saturation de l'hémoglobine fonctionnelle restante. Un taux de méthémoglobine dépassant $30 \%$ et/ou l'existence de signes d'hypoxie en dehors de la cyanose indique l'administration de bleu de méthylène à la dose de $1 \mathrm{mg} / \mathrm{Kg}$ d'une solution à $1 \%$, dans du sérum glucosé à $5 \%$ renouvelable, mais sans jamais dépasser la dose de $7 \mathrm{mg} / \mathrm{Kg}$ au risque de voir apparaître les signes de toxicité liés à l'effet méthémoglobinisant du bleu de méthylène $(2,7)$. 
Tableau I : Principaux toxiques méthémoglobinisants (3).

\begin{tabular}{|ll|}
\hline $\begin{array}{l}\text { Dérivés } \\
\text { aminés } \\
\text { et nitrés }\end{array}$ & $\begin{array}{l}\text { aminophénols, aminoquinoléines, aniline, benzidine, chloraniline, chloronitrobenzène, } \\
\text { diéthylaniline, diméthylaniline, diméthytoluidine, dinitrate (d'éthylcellosolve, d'éthylène glycol), } \\
\text { dinitrobenzène, hydrazine, nitrate (d'amyle, de propyle, de bismuth, d'éthyle, de potassium, de } \\
\text { sodium), diphénylamine, nitro (-aniline, - benzaldéhyde, -benzène, -éthane, -propane, -phénol, } \\
\text {-toluènes), phénylhydrazine, phénylhydroxylamine, quintozène, tétryl, toluidine }\end{array}$ \\
\hline $\begin{array}{l}\text { Dérivés } \\
\text { cyanés }\end{array}$ & ferricyanure, ferrocyanure \\
\hline Monoxyde d'azote \\
\hline Phénols & hydroquinone, résorcinol \\
\hline $\begin{array}{l}\text { Autres } \\
\text { toxiques }\end{array}$ & $\begin{array}{l}\text { acétanilide, chlorate de potassium et de sodium, encres à marquer le linge et teintures de } \\
\text { chaussures, fluorescéine, phénacétine, oxydant+ déficit en G-6-PD, sulfamides+ déficit en G-6-PD }\end{array}$ \\
\hline
\end{tabular}

Tableau II : Les Symptômes en fonction des taux de méthémoglobine (5).

\begin{tabular}{|cl|}
\hline $\begin{array}{c}\text { Méthémoglobinémie } \\
\% \text { d'Hb total }\end{array}$ & \multicolumn{1}{c|}{ Symptômes } \\
\hline$<15$ & Aucun \\
$15-20$ & Cyanose clinique, sang chocolat \\
$20-45$ & $\begin{array}{l}\text { Dyspnée, asthénie, vertige, céphalée } \\
\text { syncope }\end{array}$ \\
$45-55$ & Dépression nerveuse centrale \\
$55-70$ & $\begin{array}{l}\text { Coma, convulsions, insuffisance } \\
\text { circulatoire, troubles du rythme }\end{array}$ \\
& Décès possible \\
\hline
\end{tabular}

\section{Matériel et méthode}

L'étude de l'évolution des méthémoglobinémies est réalisée sur le logiciel Excel 97

Les paramètres biologiques ont été déterminés avec les méthodes suivantes :

- Gaz du sang et cooxymétrie (méthémoglobine) : Synthesis 15 u (IL France)

- LDH et autres paramètres biochimiques (fonction rénale, bilan hépatique...) : Olympus AU 600 (selon les recommandations de l'IFCC)

- Numération formule sanguine et plaquette : H.3, Technicon (Bayer France)

- G6PD : cinétique UV $340 \mathrm{~nm}$ à $30^{\circ} \mathrm{C}$ (trousse G6PD Sigma Diagnostics) sur spectrophotomètre (ABC+, Secoman France)

- Haptoglobine : Néphélémétrie sur BNA (Behring, France)
- L'analyse des constituants toxiques du produit ingéré a été réalisée au laboratoire central de la préfecture de police de Paris (lors de la première hospitalisation). Les méthodologies utilisées sont : chromatographie en phase gazeuse et par couplage chromatographie en phase-spectrométrie de masse

- A J1 (premier jour de la première hospitalisation), pour vérifier notre méthode de détermination de la méthémoglobinémie par cooxymétrie, nous avons adressé en urgence un échantillon du prélèvement correspondant à celui effectué vers la $3^{\text {enc }}$ heure (voir courbe fig. 2) au laboratoire de Biochimie Toxicologie de l'hôpital Fernand Widal à Paris. Le résultat obtenu par spectrométrie visible (méthode de référence) était similaire à celui obtenu par cooxymétrie : $4,90 \mathrm{mmol} / \mathrm{L}$ (équivaut à 58,5\% rapporté au taux d'hémoglobine de la patiente) par spectrométrie versus $55,4 \%$ par cooxymétrie.

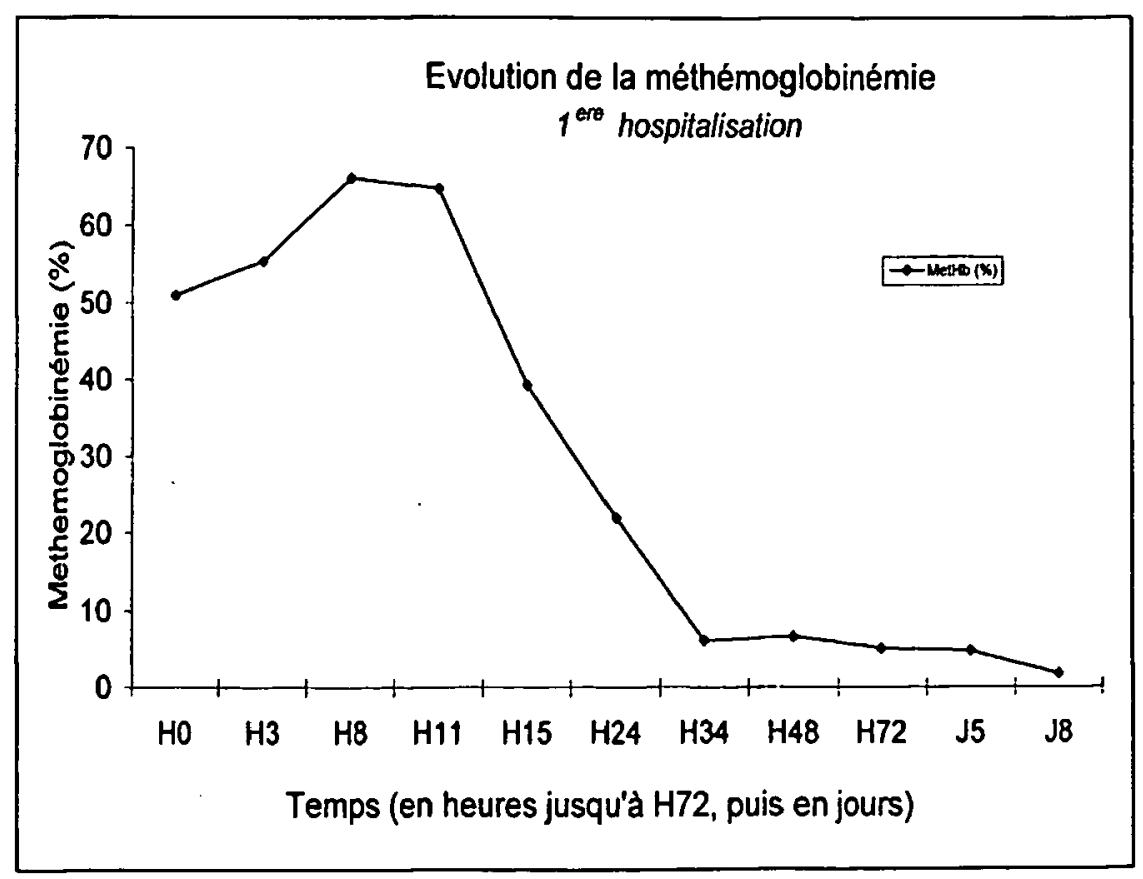

Figure 2 


\section{Observation clinique}

Une femme de 65 ans est amenée aux urgences médicales par les pompiers une heure après sa tentative de suicide par ingestion volontaire d'une teinture noire pour chaussures (quantité ingérée estimée à $200 \mathrm{~mL}$ d'une teinture de marque FAISCA de la société GIGAL au Portugal). La patiente est diabétique, hypertendue, suivie pour syndrome dépressif. Cliniquement, la patiente est somnolente, cyanosée (aspect gris ardoisé), apyrétique, sans aucun retentissement respiratoire, et un état hémodynamique normal. L'auscultation cardiopulmonaire est normale. L'examen neurologique ne retrouve aucun signe de localisation, Glasgow 12-13. Il s'agit donc d'une cyanose isolée, que n'explique pas l'état cardio-respiratoire. La teinte des téguments est à peu près généralisée mais surtout marquée à la face où elle est particulièrement intense au niveau du lobe des oreilles et des ailles du nez et même des conjonctives.

Les résultats biologiques sont les suivants : gazométrie sous $10 \mathrm{~L}$ d'oxygène : $\mathrm{pO}_{2} 209 \mathrm{~mm} \mathrm{Hg}, \mathrm{pCO}_{2} 36 \mathrm{~mm}$ $\mathrm{Hg}, \mathrm{CO}_{2} \mathrm{~T} 23 \mathrm{mmol} / \mathrm{L}$ et $\mathrm{pH} 7.44$, méthémoglobinémie $51 \%$, taux d'hémoglobine $13.2 \mathrm{~g} / \mathrm{dL}, \mathrm{GB} 1710^{3} / \mu \mathrm{L}$, plaquettes $31810^{3} / \mu \mathrm{L}, \mathrm{TP}: 68 \%$, haptoglobine : $1.9 \mathrm{~g} / \mathrm{L}$ (normale : 0,66 à 1,76 g/L), LDH : $400 \mathrm{UI} / \mathrm{L}$ (normale : 220-500 UI/L). Le bilan hépatique et la fonction rénale sont normaux. Les recherches de toxiques (antidépresseurs tricycliques, barbituriques et benzodiazépine) et de déficit en G6PD sont négatives. L'ECG et les radiographies du thorax ne montrent aucune anomalie.

Devant un tel taux de méthémoglobine, la malade reçoit un total de $6 \mathrm{~mL}$ de la solution à $1 \%$ de bleu de méthylène (ampoules unitaires de $1 \mathrm{~mL}$ ) dans les 8 heures qui suivent son admission (doses administrées dans du sérum glucosé à $5 \%$ )

L'état de la patiente ne s'améliorant pas (les taux de méthémoglobine continuent d'augmenter et la cyanose ne s'atténue pas), la patiente est alors transférée en réanimation où elle bénéficie d'une oxygénothérapie et d'un nouveau traitement au bleu de méthylène : $16 \mathrm{~mL}$ à la $11^{\text {ème }}$ heure puis $8 \mathrm{~mL}$ à la $20^{\text {eme }}$ heure (après l'admission). A J2 la méthémoglobinémie revient à la normale : $0.2 \%$ (voir fig. 2). La patiente est ensuite orientée, pour un suivi clinique et biologique, en médecine interne où elle présente, dans la semaine suivant son admission, une anémie hémolytique avec un taux d'hémoglobine à $5 \mathrm{~g} / \mathrm{dL}$ et une haptoglobine effondrée à $0,1 \mathrm{~g} / \mathrm{L}$ (normales : 0,66 à $1,76 \mathrm{~g} / \mathrm{L}$ ). L'installation brutale de cette hémolyse nécessite une transfusion sanguine de 5 culots globulaires.

Les principaux constituants identifiés au laboratoire central de la préfecture de police à Paris après analyse de la teinture sont les suivants : aniline, éthanol, métha- nol, acétate d'éthyle. L'évolution ayant été favorable et après avis du psychiatre, la malade est mise sous antidépresseurs et adressée en maison de repos pour convalescence.

Deux mois plus tard, la patiente récidive avec le même toxique. La détermination de la méthémoglobinémie est réalisée dans la demi-heure qui a suivi son admission. Le taux de méthémoglobine était de $56 \%$. L'évolution est rapidement favorable en raison de l'efficacité de la prise en charge et de la rapidité de la mise en route du traitement spécifique au bleu de méthylène. En effet une dose de départ de $10 \mathrm{~mL}$ (solution à $1 \%$ ), renouvelée 1 heure après l'admission avec une dose de $15 \mathrm{~mL}$ a ramené le taux de méthémoglobine à $16 \%$ à la $2^{\text {rme }}$ heure (voir fig. 3)

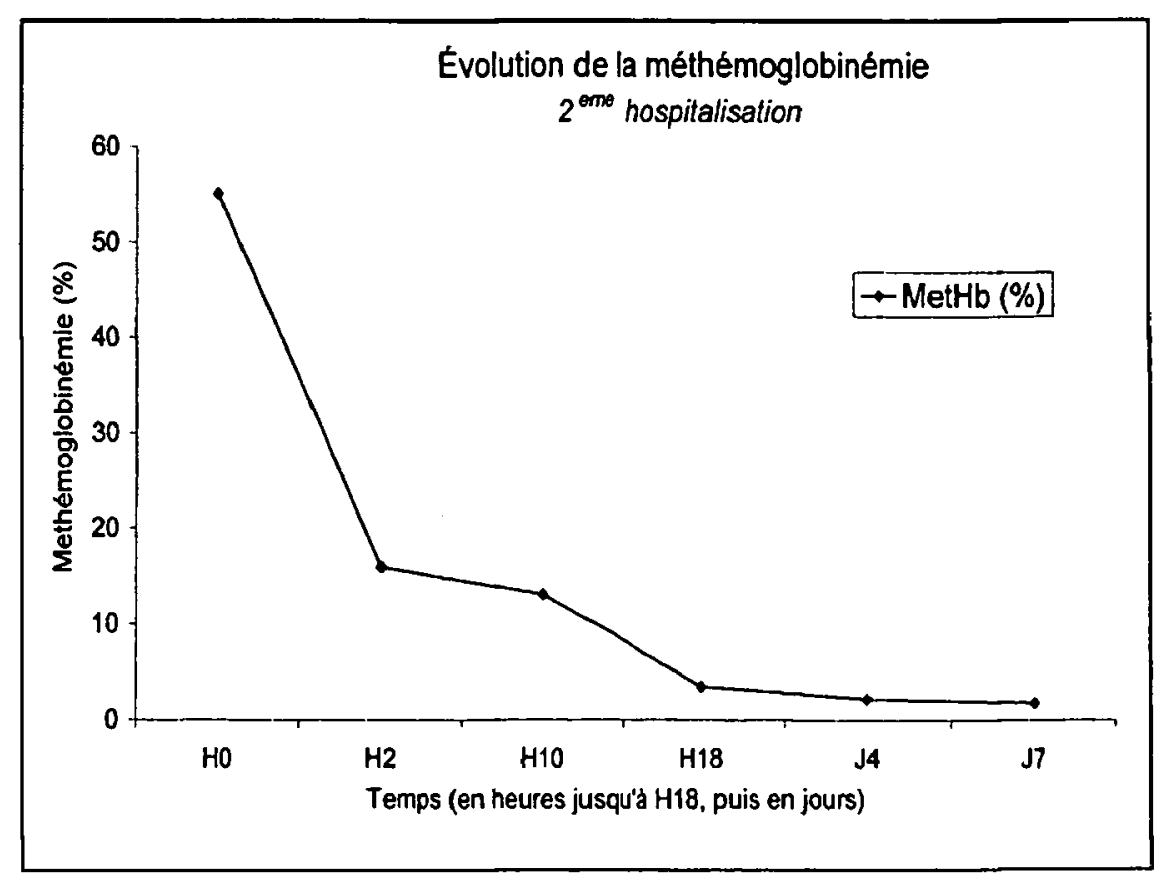

Figure 3

\section{Discussion}

Les intoxications non médicamenteuses, sont rares mais dangereuses, qu'elles soient accidentelles, suicidaires ou criminelles. La mortalité, en cas de prise aiguë, est importante, pouvant varier entre 10 et $35 \%$ (8). La mortalité de l'intoxiqué conscient est 4 fois plus importante que la mortalité du sujet comateux, car le sujet conscient et coopérant inquiète moins le médecin d'urgence, que le patient comateux (6). Le diagnostic d'une méthémoglobinémie repose sur la clinique, le dosage au laboratoire de la méthémoglobine, les circonstances de l'intoxication et l'identification du produit en cause. Dans notre cas, la cyanose a intéressé l'ensemble du revêtement cutanéo-muqueux. L'absence de toute cause cardiaque ou pulmonaire de la cyanose et l'aspect brun foncé du prélèvement ont fait évoquer la méthémoglobinémie. Le diagnostic a été confirmé au laboratoire en montrant un taux de méthémoglobine s'élevant à $51 \%$. 
La prise en charge de la patiente, au cours de la première hospitalisation en réanimation, a permis de faire régresser rapidement la cyanose et la méthémoglobinémie. En effet l'allure de la courbe d'évolution des méthémoglobinémies (fig. 2) est marquée par un pic entre la $8^{\text {eme }}$ et la $11^{\text {ème }}$ heure, malgré le début d'un traitement au bleu de méthylène dès son admission aux urgences médicales. La courbe n'entame une diminution des taux qu'après la $11^{\text {ème }}$ heure. Ceci s'explique par une dose de départ de bleu de méthylène insuffisante : $6 \mathrm{~mL}$ dans les 8 heures qui suivent son admission, $2 \mathrm{~mL}$ vers 1 heure $1 / 2$ et $4 \mathrm{~mL}$ à la $6^{\text {eme }}$ heure. L'évolution. inquiétante des taux de méthémoglobine (66\% à la $8^{\text {eme }}$ heure) et la non-régression de la cyanose a amené le réanimateur à renouveler les doses de bleu de méthylène par $16 \mathrm{~mL}$ de la solution à $1 \%$ à la $11^{\text {ene }}$.

Normalement la cyanose doit commencer à régresser dès la première heure qui suit la première injection de bleu de méthylène et le taux de méthémoglobine doit diminuer parallèlement. Ce n'est pas le cas au cours de cette première hospitalisation, car le traitement est non seulement insuffisant, mais aussi, trop étalé dans le temps.

Lors de la deuxième hospitalisation (fig. 3), la méthémoglobinémie diminue très rapidement, après l'admission (dans les deux premières heures). En effet, la malade étant connue pour ses antécédents de tentative de suicide, le produit ingéré a été rapidement identifié (même produit que lors de la première hospitalisation : teinture noire pour chaussures) et la dose de bleu de méthylène administrée a été d'emblée efficace : $10 \mathrm{~mL}$ dans la demi-heure qui a suivi son admission et $15 \mathrm{~mL}$ 1 heure après. Ce qui a fait chuter le taux de méthémoglobine à $16 \%$ dès la $2^{\text {zme }}$.

Dans le cas présenté, il s'agit probablement d'une intoxication à l'aniline contenue dans la teinture. Les dérivés $\mathrm{N}$ hydroxylés de l'aniline, après une péroxydation aboutissant à des dérivés nitrosés, sont à l'origine de la méthémoglobinémie. Un processus d'auto- aggravation s'installe car-l'organisme est capable de retransformer les dérivés nitrosés en dérivés $\mathrm{N}$ hydroxylés et e cycle recommence avec à chaque fois une production d' $\mathrm{H}_{2} \mathrm{O}_{2}$ qui nécessite la présence du Glutathion pour sa destruction. On est ainsi devant un processus pathologique entretenu par l'organisme car non seulement celui-ci fabrique le produit oxydé au niveau du foie, mais le fabrique aussi au niveau du globule rouge. Le bleu de méthylène permet la rupture de ce cycle en entraînant la réduction de la méthémoglobine avec consommation de glucose.
L'efficacité du bleu de méthylène dépend de l'intégrité du shunt des pentoses qui produit le NADPH. C'est ainsi que l'intoxication par le chlorate de sodium induisant une inactivation enzymatique de la glucose-6phosphate-deshydrogénase (enzyme clé du shunt des pentoses) explique l'inefficacité du bleu de méthylène (9).

L'hémolyse aiguë tardive rencontrée chez cette malade, lors de la première hospitalisation, une semaine après son admission (haptoglobine à $0,1 \mathrm{~g} / \mathrm{L}$ ) est probablement due à l'activité hémolytique des dérivés de l'aniline, mais certainement pas au bleu de méthylène puisque les doses administrées n'ont jamais dépassé $7 \mathrm{mg} / \mathrm{Kg}$ de poids corporel.

\section{Conclusion}

Les méthémoglobinémies acquises sévères secondaires ne cèdent pas sous oxygénothérapie. Le pronostic dépend de la rapidité de la mise en route du traitement spécifique au bleu de méthylène qu'on ne peut débuter qu'après confirmation du diagnostic par le laboratoire. En effet un taux de méthémoglobine inférieur à $30 \%$ est spontanément corrigé par la voie principale de réduction de la méthémoglobine (5), alors que des taux supérieurs à 35-40\% dépassent les capacités de réduction de l'organisme et nécessitent le traitement au bleu de méthylène. La méthémoglobinémie doit être demandée en urgence ainsi qu'un dosage de G6PD, et l'oxygénothérapie est débutée à fort débit, dans le but d'augmenter la saturation en oxygène de l'hémoglobine fonctionnelle restante.

Le cas présenté ici souligne l'intérêt du suivi rapproché des méthémoglobinémies. En effets les dosages rapprochés ont permis de détecter rapidement, l'évolution défavorable de la méthémoglobinémie, lors de la première hospitalisation, et a permis un réajustement salutaire des doses de bleu de méthylène.

Actuellement, la majorité des laboratoires hospitaliers sont équipés de cooxymétres associés ou non à un appareil à gaz du sang, et sont en mesure de rendre un résultat de méthémoglobinémie dans les 15 minutes qui suivent l'arrivée du prélèvement au laboratoire. La détermination du taux de méthémoglobine par cooxymétrie n'est pas aussi performante que la détermination par spectrophotométrie (méthode de référence), mais possède une sensibilité satisfaisante, et surtout en raison de sa rapidité d'exécution, convient parfaitement à l'urgence médicale représentée par la méthémoglobinémie grave (taux $>35-40 \%$ ). 


\section{Remerciements}

Nous remercions Monsieur Thierry LEBRICON, biologiste à l'hôpital Saint-Louis (Paris), pour sa précieuse collaboration.

Nous remercions également, le laboratoire de Biochimie et de Toxicologie de l'hôpital Fernand Widal (Paris) pour l'explication claire de la méthodologie employée dans le dosage de la méthémoglobinémie, et le laboratoire central de la préfecture de Paris pour l'analyse des constituants du toxique et l'explication détaillée des méthodologies employées.

\section{Références}

1. Galacteros F. Méthémoglobinémies et Sulfhémoglobinémies. In : L'Hématologie de Bernard Dreyfus, ed. Flammarion; $1992: 29: 407-409$

2. Danel V. Méthémoglobinémies toxiques. In : Danel V., Barriot P., Les intoxications aiguës, ed Arnette, Paris ; $1993: 49-57$

3. Bismuth C., Baud F., Conso F., Dally S., Frejaville J. P., Garnier R., Jaeger A. In : Toxicologie Clinique, $5^{\text {eme }}$ ed. Flammarion, Paris ; $2000: 43-44$

4. Harrison M. R. Toxic methaemoglobinaemia, a case of acute nitrobenzene and aniline poisoning treated by exchange transfusion. Anaesthesia $1977 ; 32: 270-272$

5. Staikowsky F., Perret A., Pevirieri F., Zanker C., Zerkak D., Pelloux P., Danhiez F. L'intoxication au "poppers", cause rare de méthémoglobinémie observée aux urgences. La Presse Médicale 1997 ; n 29 : 1381-1384
6. Delaunay L., Carli P. Cyanose. In : Carli P.-Riou B., Urgences médico-chirurgicales de l'adulte. Collection Anesthésie et réanimation d'aujourd'hui, ed Arnette 1991: 185-187

7. Le Cam Y., Carel N., Guiriec B. Méthémoglobinémie par inhalation de "poppers". A propos d'un cas. Réan Urg $1997 ; 6: 95-97$

8. Bismuth C. Intoxications non médicamenteuses. La Revue du Praticien $2000 ; 50: 361-364$

9. Ceyrat A., Thouret J. M., Clavier B., Berbineau A., Bohe J., Fournier G. Intoxication au chlorate de sodium. TOXICORAMA 1999 ; vol. XI, $\mathrm{n}^{\circ} 1: 35-39$

10. Viala A. Mécanismes et manifestations de l'action des toxiques au niveau sanguin. In : Eléments de toxicologie. Editions Médicales Internationales ; 1998 : 63-73 